\title{
Mise en évidence des effets d'une irradiation aiguë localisée par imagerie de résonance magnétique du tissu cutané
}

\author{
A. CHALANSONNET*, N. EL KAMOUNI*, A. BRIGUET*,

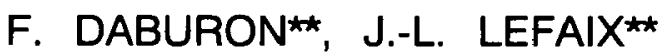

(Manuscrit reçu le 17 février 1993)

RÉSUMÉ

Des zones anatomiques superficielles exposées au rayonnement gamma d'une source d'iridium 192 ont été suivies in vivo par imagerie de résonance magnétique. Une étude de cas a été effectuée sur trois miniporcs irradiés à des doses de 96, 32 et 64 Gy à la peau, sur la région dorsale entre les vertèbres 77 et L4. Les acquisitions réalisées sur un système à 1,5 tesla montrent que les images pondérées en temps de relaxation spin-spin $\left(T_{2}\right)$ font apparaître une diminution du signal pendant les deux premières semaines suivant l'irradiation. Les variations observées, analogues dans la peau, le tissu adipeux et le muscle souscutané, sont plus fortes dans la peau. Cette observation indique que l'imagerie de résonance magnétique, qui peut être un moyen de diagnostic précoce des brûlures radiologiques en profondeur, peut également renseigner de façon spécifique et précoce sur le comportement du tissu cutané.

ABSTRACT Over-exposures with gamma rays of a 192 Ir source performed on pig skin, have been followed by magnetic resonance imaging. A group of 3 minipigs was given single doses of 96, 32 and 64 Gy applied to the back skin (between $\Pi 7$ and L4) from bottom to neck respectively. NMR images were taken from a Siemens Magnetom 63 SP system operating at 1.5 tesla. $T_{2}$-weighted spin echo images showed that signal intensities in the irradiated areas were significantly decreased during the three weeks following irradiation. The signal evolution was similar in skeletal striated muscle, subcutaneous adipose tissue and skin but with noticeably different amplitudes. The larger variation was observed on skin. This observation, which can be done in the early days after the exposure, suggests that the method could be an important aid to diagnose or to predict tissue necrosis and fibrosis.

\section{Introduction}

L'irradiation localisée de type accidentel intéresse en premier lieu la peau et les muscles sous-jacents. Les techniques d'imagerie par résonance magnétique s'avèrent être des outils adaptés au diagnostic pré-

\footnotetext{
* Laboratoire de résonance magnétique nucléaire, Université Claude Bernard, Lyon I, 43 Boulevard du 11 Novembre, 69622 Villeurbanne Cedex, France.

* Commissariat à l'énergie atomique, Direction des sciences du vivant, Département de pathologie et toxicologie expérimentales, Laboratoire de radiobiologie appliquée, CEN Saclay, 91191 Gif-sur-Yette Cedex, France.
} 
coce des lésions radioinduites [6]. Les lésions superficielles et profondes induites localement $[8,10]$ se traduisent habituellement par des modifications des temps de relaxation magnétique nucléaire qui jouent le rôle de facteur de contraste des images [15]. Après irradiation, l'apparition d'un hypersignal en profondeur, observé expérimentalement dans les muscles squelettiques de porc et de lapin a déjà pu être corrélée à la dose de rayonnement reçu $[5,11]$. Une analyse statistique des images devrait conduire de façon générale à déterminer des relations de type dose-effet susceptibles de se manifester entre l'intensité du rayonnement, la réponse des tissus vivants et la chronologie du développement des lésions. Il est donc intéressant de mettre à profit les améliorations de la sensibilité et de la résolution spatiale des systèmes d'imagerie actuels, pour s'intéresser aux phénomènes induits aux interfaces derme/tissu adipeux sous-cutané/muscle strié squelettique par une irradiation localisée. Par ailleurs, le développement des techniques d'observation par résonance magnétique nucléaire a justement offert une large place aux examens iconographiques et spectroscopiques très localisés $[1,2]$. C'est ainsi qu'il est désormais possible de réaliser des images du tissu cutané avec une résolution très inférieure au millimètre [3]. Par conséquent il devient envisageable de visualiser les variations du signal à l'intérieur même de l'épaisseur de la peau. Une grande importance est donc apportée aux méthodes capables de détecter précocement des anomalies consécutives aux irradiations accidentelles, principalement lorsque ces approches ont des possibilités diagnostiques et pronostiques.

Dans cette étude expérimentale in vivo chez le porc, nous rapportons un certain nombre d'observations effectuées sur la variation de l'intensité du signal RMN des tissus cutanés et immédiatement sous-jacents. Ces tissus ont été irradiés par une source gamma d'iridium 192 et un protocole d'observation par imagerie localisée du dos chez le miniporc a été mis au point. A l'inverse de ce qui se passe en profondeur dans le muscle irradié à forte dose où la formation d'un œdème crée un renforcement du signal RMN [5], les tissus superficiels montrent une diminution d'intensité de signal dans les premiers jours qui suivent l'irradiation.

\section{Matériels et méthodes}

\subsection{Modèle animal et technique d'irradiation}

Le modèle expérimental d'irradiation aiguë localisée chez le porc développé au CEN-Saclay a été utilisé [4]. Trois femelles miniporc Göttingen adultes de 18 mois et pesant $20 \mathrm{~kg}$ ont été soumises, sous anesthésie générale, à des irradiations localisées et collimatées sur les lombes. L'irradiateur est un appareil de gammagraphie industrielle (GR-50) renfermant une source d'iridium $192\left(E_{\gamma}=0,38 \mathrm{MeV}\right)$ avec un débit de dose de $6 \mathrm{~Gy} / \mathrm{min}$ à la peau. Le collimateur en plomb de $20 \mathrm{~mm}$ de diamètre intérieur impose une distance source-peau de $17 \mathrm{~mm}$ pendant l'irradiation [12]. Pour chaque animal, trois doses (96, 32 et $64 \mathrm{~Gy})$ 
ont été appliquées sur des zones distinctes préalablement tatouées et réparties respectivement de l'arrière vers l'avant de l'animal, entre les vertèbres L4 et T7. Le gradient de dose existant autour de la source d'iridium est tel qu'en surface $100 \%$ de la dose est délivrée, alors qu'elle n'est plus que $46 \%$ à $1 \mathrm{~cm}$ de profondeur. Ces trois doses ont été choisies pour que le gradient de doses présente une continuité permettant de couvrir la gamme de 32 à 96 Gy. Toutefois, dans la suite de cet exposé, les trois situations seront analysées séparément. Pendant le premier mois après irradiation, les expériences d'imagerie ont été réalisées en aveugle, c'est-à-dire en absence de toute symptomatologie cutanée [13].

\subsection{Expériences d'imagerie par résonance magnétique}

Les expériences d'imagerie par résonance magnétique ont été réalisées sous anesthésie générale des animaux (halothane $(2 \%)$, oxygène $(2 / 3)$ et protoxyde d'azote (1/3)). Les animaux ont été maintenus immobiles et surveillés pendant toute la durée des mesures qui demandaient environ $1 \mathrm{~h} 30 \mathrm{~min}$ par sujet. L'appareil d'imagerie employé (imageur Siemens Magnetom $63 \mathrm{SP}$ ) opérait à la fréquence de $63 \mathrm{MHz}$ pour le proton, dans un champ magnétique statique de 1,5 tesla. L'obtention d'images localisées sur le dos des animaux a nécessité une antenne réceptrice de dimensions réduites et à faible champ de vue. L'emploi de l'antenne de surface prévue pour l'imagerie de l'orbite, et dont le diamètre moyen est de $10 \mathrm{~cm}$, répondait à cette exigence. Sa sensibilité s'est avérée excellente et le choix d'une petite région d'intérêt a conduit à une résolution spatiale inférieure à $0,3 \mathrm{~mm}$. La configuration radiofréquence employée était alors celle des bobines croisées. L'antenne d'émission étant celle qui est utilisée pour les observations du type "corps entier", l'antenne de réception pouvait être fixée sur le dos de l'animal tout en étant centrée sur les trois zones irradiées. II n'était donc pas nécessaire de la repositionner au cours des observations successives réalisées sous forme de coupes transversales de $3 \mathrm{~mm}$ d'épaisseur. En déplaçant la coupe de l'arrière vers l'avant du sujet, il était ainsi possible de traverser successivement les régions ayant reçu 96,32 et 64 Gy.

\subsection{Séquences d'imagerie}

Les séquences standard d'imagerie par échos de spins, par échos de gradient et par des techniques rapides avec faible angle de basculement [7] ont été testées. Nous n'avons retenu que celles qui permettaient d'observer ou de déceler des variations d'intensité dans la région irradiée tout en bénéficiant de la meilleure résolution spatiale. Le recueil du signal en mode d'écho de spins s'est avéré le plus intéressant car il conduit à des signaux généralement plus intenses qu'avec le mode écho de gradient. Pour cette séquence qui nécessite deux impulsions radiofréquences, le temps de répétition joue un rôle important vis-à-vis du retour à l'équilibre des aimantations. Si cet intervalle de temps est court, la pondération du signal est alors gouvernée par la relaxation spin-milieu (ou relaxation longitudinale): les régions à temps de relaxation spin-milieu 
$\left(T_{1}\right)$ courts apparaissent comme les plus intenses sur l'image [15]. Lorsque le temps de répétition est suffisamment long pour permettre à la relaxation longitudinale de s'effectuer totalement, la pondération du signal dépend des temps de relaxation spin-spin: les régions les plus intenses sont celles qui présentent les temps de relaxation spin-spin $\left(T_{2}\right)$ les plus longs.

Finalement, les deux séquences retenues pour la mesure de l'intensité du signal étaient du type échos de spins :

- avec pondération en $T_{1}$ (SE $T_{1}: T_{R}=450 \mathrm{~ms}, T_{E}=15 \mathrm{~ms}$ ) où $T_{R}$ représente le temps de répétition et $T_{E}$ le temps d'écho et,

- avec deux pondérations possibles en $T_{2}\left(S E T_{2}: T_{R}=2000 \mathrm{~ms}\right.$, $T_{E_{1}}=22 \mathrm{~ms}, T_{E_{2}}=75 \mathrm{~ms}$ ).

Toutes les expériences ont été réalisées en mode multicoupe, où 30 coupes ont été effectuées à chaque observation. Les images étaient ensuite reconstruites et représentées dans un format de $256 \times 256$ pixels puis stockées sur disque optique.

\subsection{Analyse des images}

Les variations d'intensité dans l'image ont été étudiées à l'aide du logiciel de traitement standard du dispositif Magnetom de Siemens. Compte tenu du nombre élevé de coupes obtenues pour chaque animal et pour chaque type de séquence, il a été nécessaire de ramener cette analyse à un nombre de clichés plus réduit, qui ont été limités aux régions irradiées. Par ailleurs, ces coupes permettaient d'observer simultanément des régions non affectées par l'irradiation et qui, prises dans la peau, le tissu adipeux sous-cutané et le muscle squelettique, permettaient de disposer de valeurs de référence pour l'intensité du signal des tissus normaux non irradiés. Pour chaque zone irradiée à 96, 32 et $64 \mathrm{~Gy}$, trois coupes ont été retenues. Dans chacune d'elles, l'intensité du signal de le peau, du tissu adipeux sous-cutané et du muscle non irradiés, soit $P_{0}, G_{0}, M_{0}$ les valeurs respectives d'intensité du signal, ont été mesurées sur un carré de 36 pixels. Sur les mêmes coupes, l'intensité du signal correspondant à la zone irradiée - ou supposée affectée par l'irradiation compte tenu de son emplacement - a également été mesurée sur 36 pixels et en trois emplacements différents. La largeur maximale occupée par la zone de mesure est de $1,8 \mathrm{~mm}$, ce qui correspond à un pixel de $0,3 \mathrm{~mm}$ de coté. Les valeurs sont alors notées $P_{1}, P_{2}$ et $P_{3} ; G_{1}, G_{2}$ et $G_{3}$ ou encore $M_{1}, M_{2}$ et $M_{3}$ pour la peau, le tissu adipeux et le muscle irradiés respectivement. Les valeurs normalisées sont alors obtenues en combinant, pour chaque région à dose d'irradiation différente, les résultats des trois coupes par la relation, écrite ici dans le cas de la peau :

$$
\mathrm{p}=\left(\Sigma_{\mathrm{ij}} \mathrm{P}_{\mathrm{ij}} / 3 \Sigma_{\mathrm{i}} \mathrm{P}_{\mathrm{oi}}\right)
$$

où $\mathrm{i}$ représente le numéro de la coupe $(i=1,2,3)$ et $j$ celui de l'emplacement choisi arbitrairement par l'expérimentateur dans la coupe $j=1$, $2,3)$. 
Deux relations identiques donnent $\mathrm{g}$ et $\mathrm{m}$, valeurs normalisées des intensités du signal du tissu adipeux sous-cutané et du muscle irradiés et situés sous la peau. La dispersion sur la mesure des paramètres $p, g$ et $\mathrm{m}$ est obtenue par la connaissance de la dispersion sur chaque mesure indépendante (l'écart-type sur chaque valeur du signal normalisé est également reporté sur les figures 3 et 4 ).

\section{Résultats}

Alors que les changements dans les intensités locales du signal image demandent à être mis en évidence par une étude systématique, l'ampleur de certaines modifications peut être nettement visible sur les clichés obtenus (fig. 1 et 2). La dynamique des effets ainsi observés puis quantifiés apporte des données supplémentaires concernant l'amplitude des variations du signal et leur cinétique (fig. 3 et 4 ). Les valeurs obtenues correspondent aux observations réalisées pour chaque dose $32 \mathrm{~h}$, 1 semaine, 2 semaines et 3 semaines après l'irradiation.

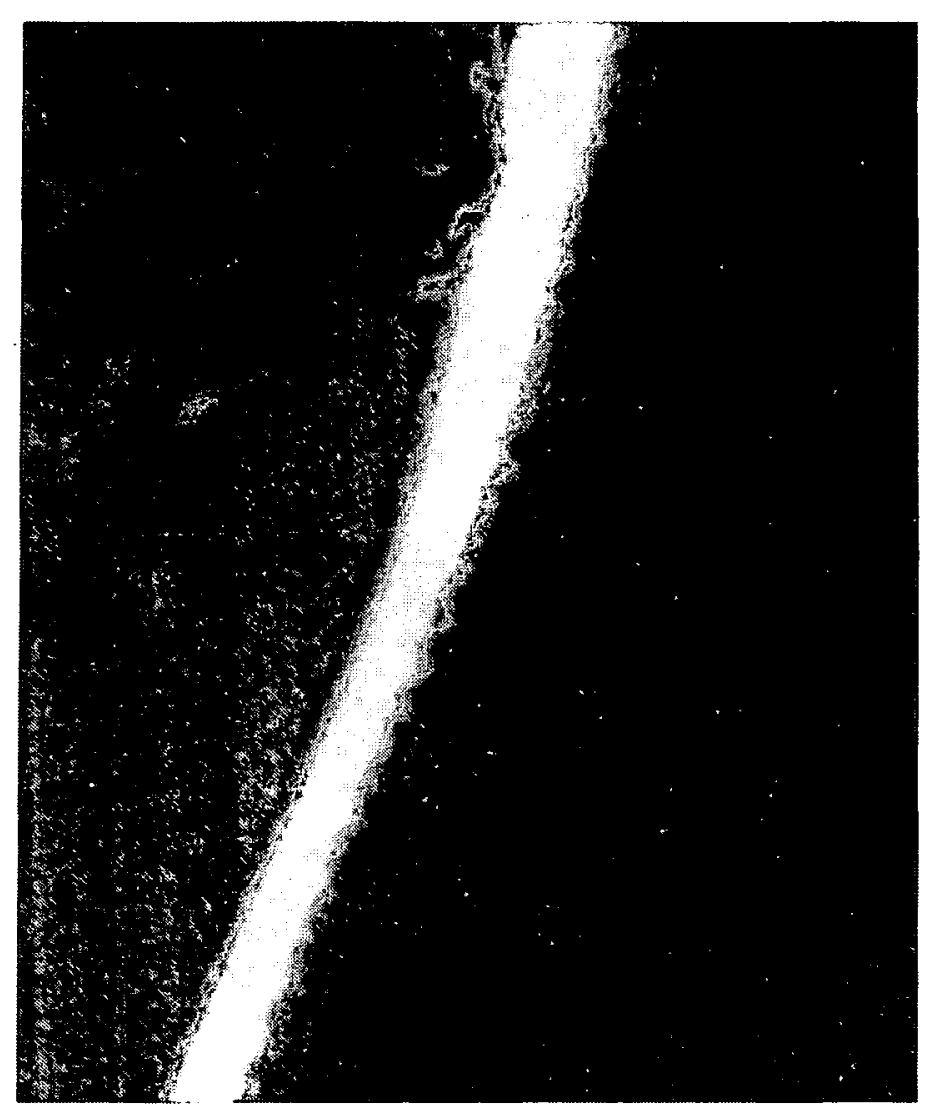

Fig. 1. - Image de tissu non irradié du dos, sur laquelle les couches successives de peau, de tissu adlpeux sous-cutané et de muscle lllosplnal peuvent être observées (cas $n^{\circ}$ 1031).

Non-exposed tissue from the back; the successive skin layers, subcutaneous adipose tissue, Iliosplnal muscle can be observed (case 1031). 


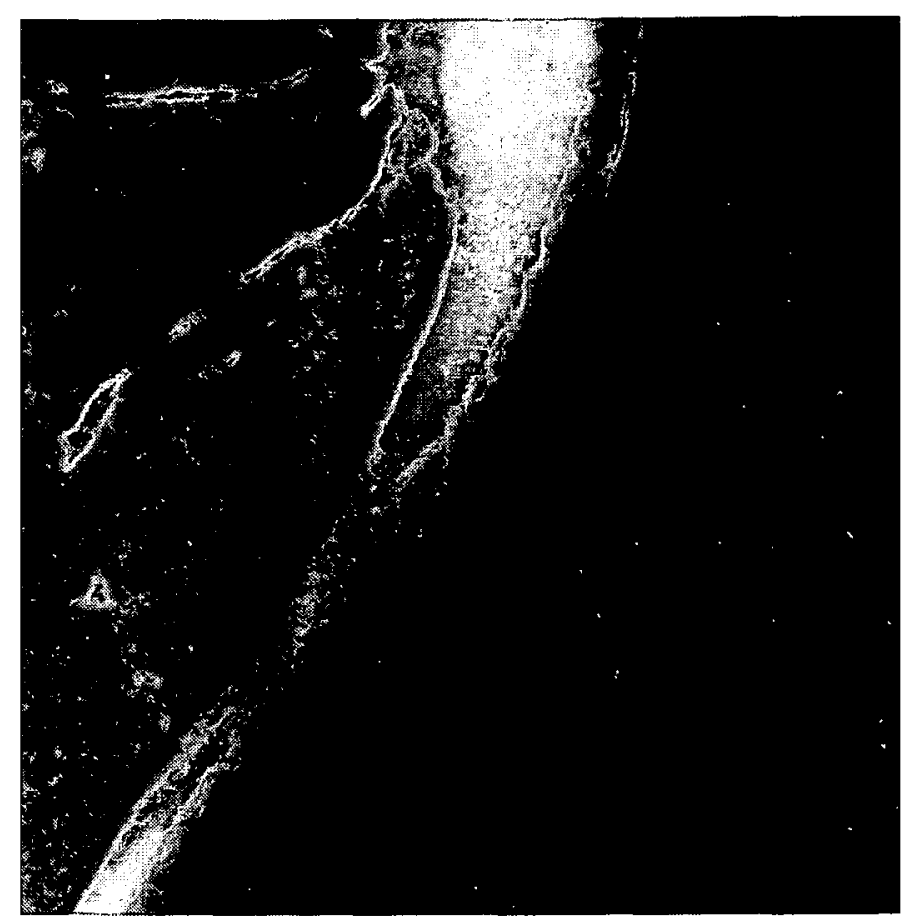

Fig. 2. - Différenciation entre zone irradiée sur la peau et zone non affectée par l'atténuation du signal, 1 semaine après une dose de 64 Gy (cas $n^{\circ} 1031$ ).

Dlfferentlation by signal attenuation between exposed skin area and unaffected area, 1 week after 64 Gy exposure (case 1031).

Ces histogrammes montrent que les valeurs normalisées de départ du signal peuvent être supérieures à 1 pour la peau alors qu'elles sont voisines de 1 pour le tissu adipeux et le muscle squelettique. Par ailleurs, la diminution relative du signal est la plus forte dans la peau comparativement aux deux autres tissus immédiatement sous-jacents, le tissu adipeux étant le milieu dans lequel le phénomène est le moins accentué. Les comportements du tissu adipeux et du muscle squelettique au premier écho $(22 \mathrm{~ms})$ et au deuxième écho $(75 \mathrm{~ms})$ sont très similaires. Un régime limite de l'intensité du signal s'installe au cours des 3 semaines après irradiation, pendant lesquelles le signal décroît. Ce régime est atteint plus rapidement dans le cas de la peau, ce qui se traduit par une variation moins nette à long terme du signal de la peau au deuxième écho.

Ces histogrammes montrent également que les variations de signal de plus forte amplitude sont systématiquement associées à la dose de 64 Gy et qu'elles conduisent à une valeur limite située au-dessous des valeurs limites observées avec 32 Gy et 96 Gy, trois semaines après irradiation.

Cette analyse a mis en évidence une diminution du signal dans les deux séquences d'échos de spins pondérées en temps de relaxation spin-spin, de manière équivalente chez les trois animaux. 


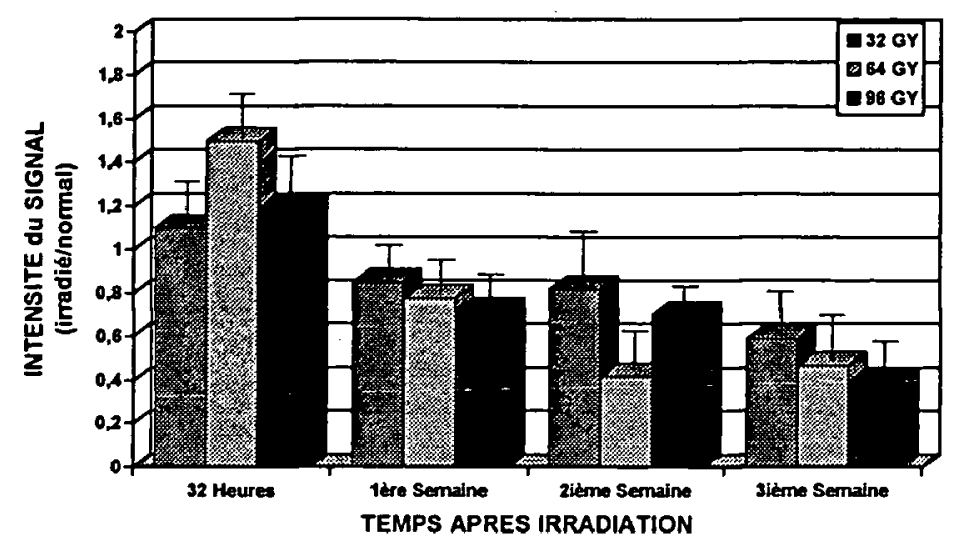

b-TISSU ADIPEUX SOUS CUTANE (1eI ECHO )

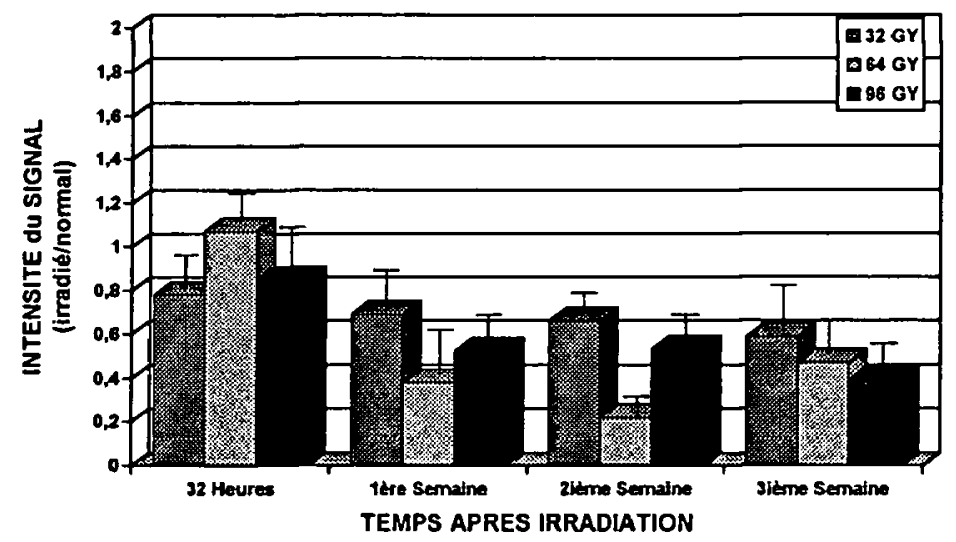

C-PEAU (1er ECHO)

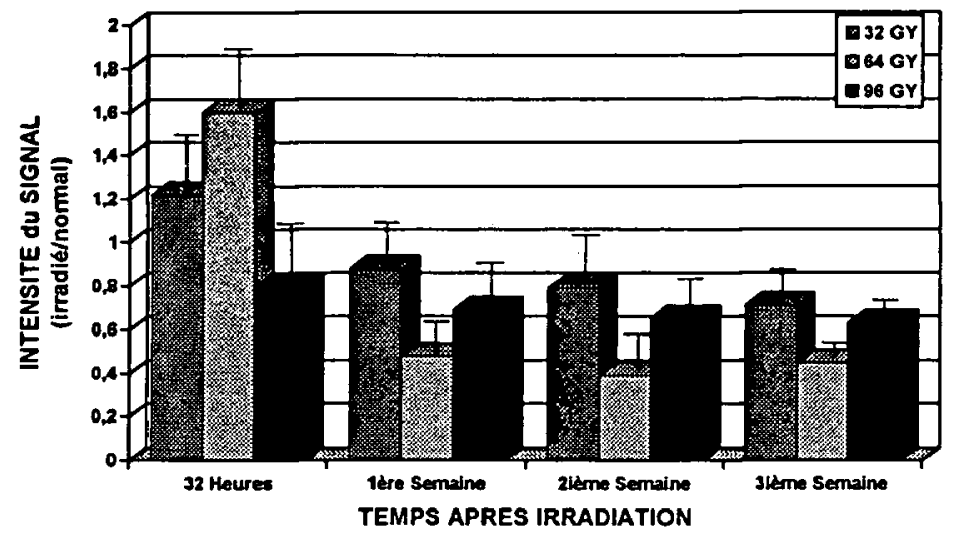

Fig. 3. - Séquence d'échos de spins pondérée en temps de relaxation spin spin $\left(T_{R}=2000 \mathrm{~ms}\right.$, $T_{E}=22$ ms). Varlatlons au cours du temps des intensités normallsées du signal (a) dans la peau, (b) dans le tissu adipeux, (c) dans le muscle squelettique d'un sujet pour les trois doses d'Irradiatlon (96, 32 et $64 \mathrm{~Gy}$; cas $\left.n^{\circ} 1031\right)$. Les premlères observations sont effectuées $32 \mathrm{~h}$ après irradlation.

$T_{2}$-weighted spin echo Images $\left(T_{R}=2000 \mathrm{~ms}, T_{E}=22 \mathrm{~ms}\right)$. Time variations of normaIIsed signal intensities for the 3 doses (96, 32, $64 \mathrm{~Gy}$ ) in : (a) skin ; (b) adipose tissue; (c) skeletal muscle. The first observations were made $32 \mathrm{~h}$ after exposure (case 1031). 
a-MUSCLE SQUELETRQU (2lème ECHO)

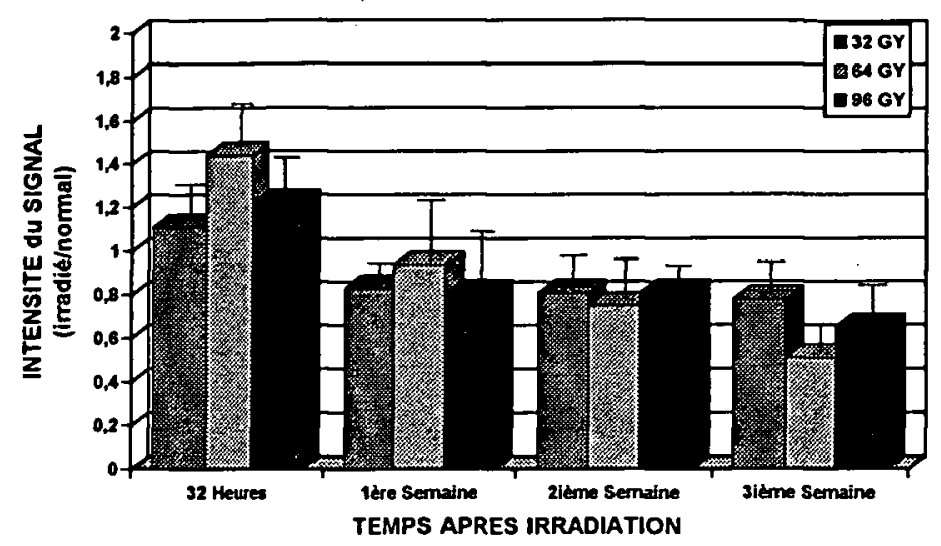

b-IISSU ADIPEUX SOUS CUTANE (2Ième ECHO)

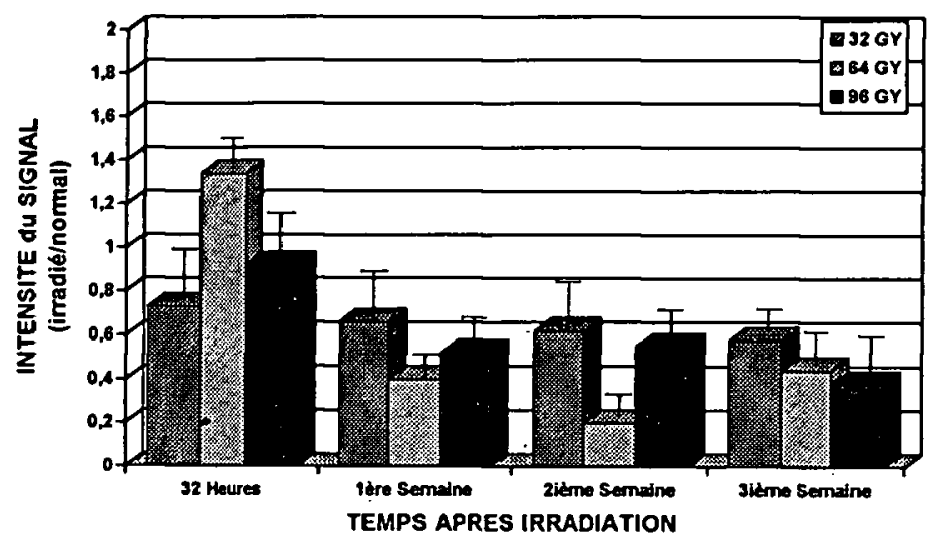

C-PEAU (2lème ECHO)

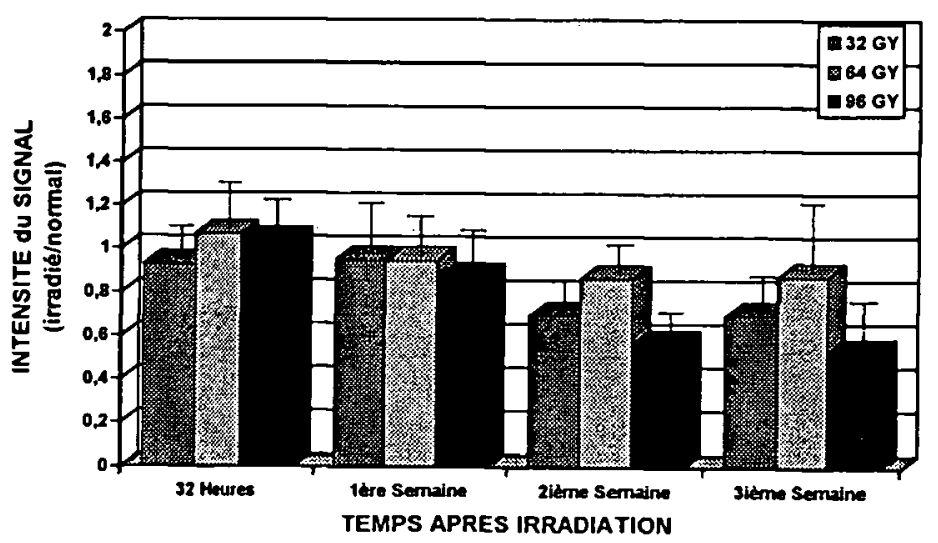

Fig. 4. - Séquence d'échos de splns pondérée en temps de relaxation spin spin $\left(T_{R}=2000 \mathrm{~ms}\right.$, $T_{E}=75 \mathrm{~ms}$, deuxlème écho). Varlations au cours du temps des Intensités normalisées du signal (a) dans la peau, (b) dans le tissu adipeux, (c) dans le muscle squelettique d'un sujet pour les trois doses d'irradiation (96, 32 et $64 \mathrm{~Gy}$; cas $\left.n^{\circ} 1031\right)$. Les premières observations sont effectuées $32 \mathrm{~h}$ après irradiation.

$T_{2}$-weighted spin echo images $\left(T_{R}=2000 \mathrm{~ms}, T_{E}=22 \mathrm{~ms}\right)$. Time variations of normaIised signal Intensities for the 3 doses (96, 32, $64 \mathrm{~Gy}$ ) in : (a) skin ; (b) adlpose tissue ; (c) skeletal muscle. The first observations were made $32 \mathrm{~h}$ after exposure (case 1031). 


\section{Discussion}

Les observations réalisées avec ces deux types de mesure sont tout à fait en cohérence et permettent de conclure à une diminution du temps de relaxation spin-spin $\left(T_{2}\right)$. En effet, la baisse d'intensité du signal ne semble pas devoir correspondre à une diminution de la concentration en protons observables et les.images pondérées en temps de relaxation spin-milieu $\left(T_{1}\right)$ ne montrent aucune variation significative d'intensité du signal. Par ailleurs, une diminution du nombre de protons observables devrait s'accompagner d'un raccourcissement de la valeur de $T_{1}$ qui compenserait exactement la diminution consécutive de l'intensité de l'image. Dans ce cas, les effets observés devraient être différents dans la peau, le tissu adipeux sous-cutanés ainsi que dans le muscle. En fait, le signal observable du tissu adipeux provient essentiellement des protons des lipides dont les fonctions sont très différentes de celles des protons de l'eau intramusculaire ou intracutanée. II semble donc peu probable que la variation d'intensité reflète la traduction immédiate et précoce de la nécrose tissulaire. Par ailleurs, l'absence d'augmentation du signal dans le cas des images pondérées par la relaxation spin-spin, augmentation attendue en raison de la possibilité de formation d'un œdème, est à noter. Nous pouvons donc retenir l'hypothèse d'une désorganisation de l'architecture macromoléculaire des tissus par le rayonnement gamma: en présence des hétérogénéités de structure, le temps de relaxation transversal effectif voit alors sa valeur locale diminuer sous l'effet des variations de susceptibilité magnétique liée à un désordre physique du milieu. Cette hypothèse pourrait être vérifiée sur des fantômes chimiques, homogènes et/ou hétérogènes, irradiés et observés dans les mêmes conditions [14] ou bien irradiés directement dans l'aimant pendant les acquisitions RMN. Par ailleurs, il n'est pas exclu que les modifications chimiques consécutives à l'irradiation puissent jour un rôle dans la recomposition des tissus et ainsi influer sur le contraste des images. Cette voie, qui peut renseigner sur la thérapeutique à suivre, demande à être explorée par spectroscopie [9].

Dans notre modèle expérimental d'irradiation collimatée, les doses d'irradiation utilisées induisent des lésions cutanées tardives dans une chronologie bien établie. En effet, après une dose de $32 \mathrm{~Gy}$, deux vagues érythémateuses apparaissent entre la $8^{\mathrm{e}}$ et la $12^{\mathrm{e}}$ semaine après irradiation sans lésion ultérieure, aussi bien au plan superficiel qu'au plan musculaire sous-cutané. Après une dose de $48 \mathrm{~Gy}$, absente de cette série expérimentale, une réaction érythémateuse s'installe entre la $8^{e}$ et la $12^{\mathrm{e}}$ semaine après irradiation, suivie d'une phase d'épidermolyse sèche qui se développe à partir de la $12^{\mathrm{e}}$ semaine, sans radionécrose cutanéo-musculaire tardive. Pour les doses de 64 et 96 Gy, après un érythème fugace dans les premières heures, un érythème soutenu s'installe entre la $3^{e}$ et la $5^{e}$ semaine suivi d'une période d'épithéliite exsudative entre la $7^{\mathrm{e}}$ et la $12^{\mathrm{e}}$ semaine après irradiation. A ces deux doses d'exposition, le processus de dégradation cutanéo-musculaire abouti à une fibrose musculaire tardive dont le volume est dépendant de la dose 
d'irradiation [13]. En raison de cette chronologie tardive, nous pouvons considérer que, pour la dose de $32 \mathrm{~Gy}$, la modification du signal n'est pas observable dans les trois premières semaines après irradiation. Pour la dose de 64 Gy et aux temps d'observation choisis dans ce travail, les effets présentent l'amplitude maximale. La peau microvascularisée subissant un apport rapide de fluide, qui traduit l'érythème précoce, est ensuite le siège d'une modification des protéoglycanes suivie d'une dégradation progressive du derme et de l'hypoderme, à laquelle les images permettent d'assister. Pour la dose de $96 \mathrm{~Gy}$, nous pouvons envisager que la même réaction a déjà eu le temps de se développer dans la peau au cours des $32 \mathrm{~h}$ précédant la prise des images $R M N$. A l'inverse, le tissu adipeux moins vascularisé que le derme et l'hypoderme, ne montre que la désorganisation du milieu par les radicaux libres libérés pendant l'irradiation, notamment la peroxydation des chaînes lipidiques. Une observation sélective de ce tissu en spectroscopie proton permettra de suivre les variations des concentrations relatives eau/lipide après irradiation, par rapport au tissu normal adjacent.

\section{Conclusion et perspectives}

L'analyse de l'intensité du signal dans des zones localisées d'images de résonance magnétique de tissu cutané irradié a montré que le comportement des tissus était analogue in vivo sur les trois miniporcs utilisés. Dans notre modèle expérimental, les effets les plus marqués ont été observés après une dose de 64 Gy. L'imagerie par RMN apparaît ainsi comme une technique de détection précoce des désordres cutanés induits par une irradiation localisée, puisqu'une variation significative du signal apparaît dès les premiers jours. Cependant, bien que cette étude ait été réalisée sur un modèle bien maîtrisé d'irradiation localisée $\gamma$, de type accidentel, l'irradiation par émetteurs $B$ de différentes énergies pourra conduire à une étude plus sélective in vivo du comportement du tissu tégumentaire. Par ailleurs, la recherche de relations dose-effet à travers la modification du signal pourra s'appuyer sur des études de dosimétrie RMN sur gels irradiés [14].

Remerciements : Cette étude a bénéficié du soutien d'EDF (convention $n^{\circ} 1$ G5280) D287). Nos remerciements vont également à Mme Claire Porcher pour sa conduite des opérations d'imagerie, à $M$. Jean-Jacques Leplat pour les soins portés aux animaux, ainsi qu'aux Prof. M. Amiel, J.C. Froment et $D$. Revel pour la mise à disposition de l'installation $d^{\prime} I R M$ du site Neuro-Cardiòlogique de Lyon. 


\section{RÉFÉRENCES}

[1] ACKERMAN J.J.H., GROVE T.H., WONG G.G., GADIAN D.G., RADDA G.K. Mapping of metabolites in whole animal by 31P NMR using surface soils. Nature, 1980, 283, 167-170.

[2] AXEL L. - Surface coil imaging. J. Comput. Assist. Tomogr., 1983, 8, 381-384.

[3] BITTOUN J., SAINT JALMES H., QUERLEUX B., DARRASSE L., JOLIVET O., IDYPERETTI I., WARTSKI M., RICHARD S., LEVEQUE J.L. - In vivo high resolution MR imaging of the skin in a whole body system at 1.5 tesla. Radiology, 1990, $176,457-460$.

[4] DABURON F., LEFAIX J.-L., REMY J., FAYART G., TRICAUD Y. - Intérêt et limites des mesures thermographiques microondes pour le diagnostic et le pronostic des irradiations aiguës localisées chez le porc. Radioprotection, 1985, 20 (3), 207-225.

[5] DABURON F., LEFAIX J.-L., LAVAL M., MARTIN J.-L., LE BAS J.-F., WAKSMANN B. - Intérêt de la tomographie $X$ et RMN pour le diagnostic précoce des irradiations localisées. Etude expérimentale chez le porc. J. Biophys. Biomec., 1986, 10, 89-92.

[6] DABURON F., LEFAIX J.-L., HOFFSCHIR D., FAYART G. - Dosimétrie biologique des irradiations aiguës localisées. Radioprotection, 1991, 26, suppl. 1; 265-282.

[7] HAASE A., FRAHM M., MATTHEI D., HANICKE W., MERBOLT D. - FLASH imaging: rapid NMR imaging using low flip pulses. J. Magn. Resonance, 1986, 67, 258-266.

[8] HOPEWELL J.W. - The skin, its structure and response to ionizing radiations. Int. J. Radiat. Biol., 1990, 57, 751-773.

[9] KIM Y.H., ORENBERG E.K., FAULL K.F., WADE-JARDETZKY N.G., JARDETZKY O. - 1H NMR spectroscopy: an approach to evaluation of diseased skin in vivo. J. Invest. Dermatol., 1989, 92, 210-216.

[10] LEFAIX J.-L., VEROLA O., DABURON F., BROCHERIOU C. - Les lésions cutanées et musculaires après irradiation aiguë chez le porc. Ann. Pathol., 1985, 5, 249-258.

[11] LEFAIX J.-L., DABURON F., MARTIN J.-L., JEANDEY C. - Intérêt de l'analyse en composantes principales appliquée à l'imagerie RMN pour le diagnostic précoce de l'irradiaton aiguë localisée chez le lapin. Innov. Technol. Biol. Med., 1988, 9, 429-444.

[12] LEFAIX J.-L., DABURON F. FAYART G. - Dosimetry study of an accidental overexposure to 192 gamma rays. Health Phys., 1992, 63 (6), 692-694.

[13] LEFAIX J.-L., MARTIN M., TRICAUD Y., DABURON F. - Muscular fibrosis induced after pig skin irradiation with single doses of Ir $192 \gamma$-rays. Brit. J. Radiol., 1993, 66, 537-544.

[14] OLSSON L.E., APPLEBY A., SOMMER J. - A new dosimeter based on ferrous sulfate solution and agarose gel. Apṕl. Radiat. Isot., 1991, 42, 1081-1086.

[15] WEHRLI F.W., MCFALL J.R., SHUTTS D., BREGER R., HERFKENS R.J. Mechanisms of contrast in NMR imaging. J. Comp. Assist. Tomogr., 1983, 8, 331-340.

[16] WEHRLI F.W., McFALL J.R., GLOVER G.H., GRIGSBY N., HAUGHTON V., JOHANSSON. - The dependence of nuclear magnetic resonance image contrast on intrinsic and pulse sequence timing parameters. J. Magn. Reson. Imaging, 1984, 2, 3-16. 